\title{
New aspects of the treatment of alopecia areata
}

\author{
Ligia Brzezińska-Wcisłoํㅜ, Beata Bergler-Czop¹, Dominika Wcisło-Dziadecka², Anna Lis-Święty
}

${ }^{1}$ Dermatology Department, Medical University of Silesia, Katowice, Poland

Head of Department: Prof. Ligia Brzezińska-Wcisło MD, PhD

${ }^{2}$ The Institute of Structural Research of Skin, Cosmetology Department of Medical University of Silesia, Katowice, Poland

Head of Department: Krzysztof Jasik PhD

Postep Derm Alergol 2014; XXXI, 4: 262-265

DOI: $10.5114 /$ pdia.2014.40923

\begin{abstract}
Alopecia areata (AA) is a disease involving non-scarring hair loss determined by autoimmune disorders and inflammation. The disease affects hair on the scalp and/or other parts of the body. The AA occurs in people of all ages and affects $1-2 \%$ of humans. The purpose of this paper is to present the latest knowledge on the treatment of AA. The decision on the type of treatment depends on the type of hair loss, extent of changes, general health status, the patient's age, and his/her motivation. Treatment methods should be chosen individually for each patient.
\end{abstract}

Key words: alopecia areata, treatment.

Alopecia areata (AA) is a disease involving non-scarring hair loss determined by autoimmune disorders and inflammation. The disease affects hair on the scalp and/ or other parts of the body. The AA occurs in people of all ages and affects $1-2 \%$ of humans. Clinical forms of $A A$ include [1, 2]:

- alopecia areata focalis - hair loss occurs in patches on the scalp or on other parts of the body (e.g. face, abdomen, extremities),

- alopecia areata totalis - the loss of all hair on the scalp (including eyebrows and eyelashes),

- alopecia areata universalis - the loss of all or almost all body hair,

- alopecia maligna - is a generalized long-term loss of hair, resistant to treatment,

- ophiasis or alopecia areata marginata - snake-shaped hair loss around the circumference of the head in the temporal, occipital and frontal areas,

- ophiasis inversus - the inverse pattern of hair loss, which expands from the central to the marginal area of the head,

- alopecia areata diffusa or alopecia areata reticularis diffuse or reticular hair loss where no separate bald patches can be distinguished.

Nails are affected in about $7-60 \%$ of patients. Aberrations include: koilonychia, trachyonychia, Beau lines, onychorrhexis, nail pitting, onychomadesis, onycholysis and haemorrhagic spotting of the lanula.

Poor prognostic factors include bald patches persisting for more than 1 year, aggravation or onset of hair loss before puberty, positive family history of AA, ophiasis pattern of involvement, associated nail changes, atopy, and Down syndrome [1].

Typical pathological changes are manifested as a well-separated bald spot. Skin in bald patches may be slightly depressed due to reduced mass of hair follicles, and mild erythema may develop in some cases. There is no atrophy, and on the margins, exclamation mark hair (thick and broken off) is present. Regrowing hair may demonstrate pigment alteration or a change in texture (i.e. straight or curly) [2].

Because the aetiology of AA remains unknown, the treatment is symptomatic and does not prevent disease relapse. The efficacy of many treatment methods has been questioned by many scientific authorities due to the lack of reliable clinical studies (the possibility of spontaneous hair regrowth and very few double-blind placebo-controlled clinical studies). The decision on systemic treatment depends on the type of hair loss, the extent of changes, general health status, the patient's age and motivation, and concomitant diseases. Treatment methods should be chosen individually for each patient.

Address for correspondence: Prof. Ligia Brzezińska-Wcisło MD, PhD, Dermatology Department, Medical University of Silesia, 20/24 Francuska St, 40-027 Katowice, Poland, phone: +48 3225611 82, fax: +48 32256 11 82, e-mail: kikderm@sum.edu.pl Received: 6.05.2013, accepted: 20.08.2013. 
The disease course is difficult to predict because spontaneous remissions are frequently observed, while in about $5 \%$ of cases the disease progresses into total alopecia, and in $1 \%$ of cases into universal alopecia [3, 4]. After completed treatment patients must be informed about the high risk of disease relapse [5].

In emotionally unstable patients, each exacerbation of hair loss symptoms may lead to a depression phase. In patients with psychoneurotic disorders, increased hair loss is an underlying symptom of psychopathic personality. In frequent cases, patients may have difficulties in interpersonal relations at the workplace [4] or in their private life (withdrawal from active social life).

Alopecia areata is diagnosed based on trichoscopy, the hair pull test and trichogram. Histopathological examination can be carried out if diagnosis is uncertain. Trichoscopy is a modern method and is very useful in the monitoring of treatment for AA and the evaluation of its efficacy. This is a non-invasive, easy to use and painless test, which enables the objective assessment of disease activity. Patients need no hair shaving or dying, images can be recorded, and the only requirement is the considerable experience of the operator.

The purpose of this paper is to present the latest knowledge on the treatment of AA.

Systemic glucocorticosteroids have been used in the treatment of AA for many and in different modalities. Generally, they offer better outcomes in patients with multifocal AA than in patients with other forms of the disease.

Turkish scientists [6], in their paper on pulse methylprednisolone therapy for the treatment of extensive $A A$, suggested that therapy might be an option for severe multifocal $A A$, but in alopecia totalis or universalis treatment results are unsatisfactory. The study was carried out on 15 adult patients who received methylprednisolone of $500 \mathrm{mg}$ intravenously on 3 consecutive days monthly for 3 months. According to these researchers, pulse methylprednisolone therapy appears to be a safe treatment option.

Staumont-Salle et al. carried out a 10-year-long assessment of pulse methylprednisolone therapy for AA [7]. The study included 60 patients treated between 1995 and 2000 and confirmed the low efficiency, both shortand long-term, of this treatment for AA.

Researchers from Saudi Arabia [8] evaluated the efficacy and safety of methylprednisolone for severe therapy-resistant $A A$, and found that the use of this drug in severe forms of AA has relative efficacy and tolerance, but with a high relapse rate.

In 2011, Alsantali presented a new treatment plan for AA in Clinical Cosmetic Investigative Dermatology. According to this plan, glucocorticosteroids are a third-line option in the treatment of AA. This includes: a once-monthly pulse of $300 \mathrm{mg}$ methylprednisone (or a once-monthly pulse of $200 \mathrm{mg}$ ), intramuscular triam- cinolone acetonide of $40 \mathrm{mg}$ once monthly or oral dexamethasone of $0.5 \mathrm{mg} /$ day [9].

Some researchers from Iran concluded that the use of methotrexate (15-25 mg once a week for 3 months) alone or with low doses of glucocorticosteroids or azathioprine alone is an effective treatment in patients with severe and chronic AA. This therapy may be an alternative option in the treatment of patients with moderate to severe forms of AA (due to safety and efficacy). The study included 20 patients who had a 6-month-long history of hair loss. Patients received $2 \mathrm{mg} / \mathrm{kg}$ body weight of azathioprine for 6 months. Hair regrowth was achieved in $53 \%$ of patients and the drug was well tolerated [10].

Droitcourt et al. treated patients suffering from severe $A A$ using high-dose pulse therapy with methylprednisolone $(500 \mathrm{mg}$ intravenously per day for 3 consecutive days monthly over 3 months) plus $15 \mathrm{mg}$ of methotrexate once a week for the same treatment period [11]. Of the 14 patients (20 patients were treated), 10 had total hair regrowth and 4 had incomplete but satisfactory regrowth. Treatment was well-tolerated.

The analysis of many research papers has revealed a general controversy about the administration of glucocorticosteroids. Most researchers reported that the therapeutic outcome is short-lasting and the withdrawal of medication is followed by hair loss.

According to Miteva and Tosti [12], better therapeutic outcomes have been observed when treatment begins before the end of 3 months following the onset of the first symptoms in patients with universal alopecia, and before the end of 4 to 6 months in patients with focal AA. Pulse therapy based on the very short administration of high doses of glucocorticosteroids is associated with a lower number of adverse effects. Such therapy should be considered in adults with total or universal alopecia resistant to topical treatment or phototherapy [5].

Bhat et al. [13] assessed levels of trace elements (zinc, copper and magnesium) in patients with AA. Fifty patients were studied. Samples were analyzed using atomic absorption spectrometric methods. Serum zinc levels were decreased in AA patients compared to healthy controls. Serum copper and magnesium levels showed an insignificant rise.

The combination therapy (PUVA with 20 mg prednisone) may modify the immune system and increase the number of Treg cells, resulting in hair regrowth in patients with AA [12]. Hair regrowth was achieved in 100\% of patients and $22 \%$ of patients had a relapse of hair loss 3 months after the termination of the treatment. Authors emphasized the efficacy of this method mainly in patients with AA resistant to treatment.

Limitations on the use of cyclosporine in the treatment of AA result from the high relapse rate after the termination of therapy, the need for long-term drug administration, and adverse effects, e.g. nephrotoxicity [5]. 
Ito et al. [14] concluded that spontaneous remission occurs in $80 \%$ of patients with AA within 1 year, and not all patients require intense therapy, and therefore watchful observation is one of the therapeutic options. However, when hair loss is progressing, treatment becomes necessary, and pulse methylprednisolone should be preferred.

Korean scientists studied the effects of intradermal botulinum toxin injections on AA (3 times on each side of the head). One patient reported aggravation, and the remaining patients had no local improvement. These results suggest that botulinum toxin injections are not useful as a treatment in AA [15].

Some scientists from Iran claim that antidepressant treatment may result in improvement in local conditions in patients with AA who have depressive disorders [16].

Other scientists claim that hypnotherapy may be effective in achieving a significant improvement and maintaining good emotional status and life quality in patients with resistant $A$ A.

The use of biologics in the treatment of AA was also tested but no significant efficacy was shown, and there are some reports on aggravation during therapy [17].

Luk et al. [18] studied the efficacy and safety of diphenylcyclopropenone (DPCP) in Chinese patients with AA resistant to treatment with steroids and reported a good response achieved in over $50 \%$ of treated subjects.

Campuzano-Maya, of Colombia, described the case of a 43-year-old patient with an 8-month history of AA of the scalp and beard. The urea breath test confirmed Helicobacter pylori infection. The patient went into remission from AA after $H$. pylori eradication [19].

There were also attempts to treat patients with $A A$ with exposure to narrow-UVB to the medium total dose of $63.9 \mathrm{~J} / \mathrm{cm}^{2}$ or bexarotene gel, and different regrowth was achieved [20, 21].

There are also reports describing the effects of locally administered vitamin $D$ analogues in the treatment of $\mathrm{AA}$, and a comparative study on local treatment with $0.05 \%$ clobetasol and $1 \%$ pimecrolimus, where similar efficacy was found for both products [22].

Researchers from Iran determined the efficacy of topical triiodothyronine in patients with patchy AA. Ten patients with AA were treated with topical triiodothyronine and placebo applied twice daily. Hair regrowth was evaluated every 4 weeks. Blood count, along with thyroid function (T3, T4 and TSH) and liver function were also tested. After 12 weeks of treatment, there was no statistically significant difference between the outcome in the active treatment and placebo groups. Triiodothyronine was safe but not more effective than placebo [23].

Literature data on the effects of sulphasalazine on the treatment of AA can be compared with the efficacy of cyclosporine alone. Sulphasalazine is an efficient drug in cases resistant to other treatment methods, but offers a much poorer outcome in patients who had disease onset in childhood [5].

In some patients there is a clear correlation between AA and the nervous system function, and bald patches may occur suddenly after severe emotional trauma, both in children and adults. According to Manolache et al., the most frequent stressful events include the beginning of school or pre-school education, exams, change of school/group, problems with teachers, intensive studying, social problems with peers, death in the family, family financial problems, emigration of parents for work reasons, concomitant diseases and surgical procedures [24].

In addition, Willemsen et al. carried out a study to investigate whether adult AA is associated with childhood or total lifetime traumatic events. The study was carried out using the "Traumatic Experiences Checklist" [25]. Ninety patients and 91 people from the control group were surveyed. The study demonstrated that the frequency of AA is affected by lifetime traumatic events. Also, there was an increased history of childhood trauma in patients with AA compared with control subjects.

Alsantali [9], in his paper published in Clinical Cosmetic Investigative Dermatology, presented new treatment options for AA. First-line therapies include intralesional triamcinolone acetonide injections $(2.5-10 \mathrm{mg} / \mathrm{ml}$, maximum volume of $3 \mathrm{ml}$ in a single injection, repeated at 4-6 weekly intervals). The drug can also be administered using mesotherapy multi-injectors. Other treatment options include topical corticosteroids (creams, gels, ointments, lotions, and foams), followed by minoxidil, anthralin, topical immunotherapy, prostaglandin analogues, topical retinoids and capsaicin [9]. Second-line therapies include oral sulfasalazine of $500 \mathrm{mg}$ twice daily at the start, then $1 \mathrm{~g}$ twice daily for 1 month, and then $1 \mathrm{~g}$ three times daily for 3 months, PUVA, PUVA-turban, excimer laser and fractional photothermolysis laser. Thirdline therapies include systemic corticosteroids (pulse doses), methotrexate 15-25 mg/weekly for 3 months, cyclosporine A, azathioprine, biologics and psychological support. Other therapies mentioned by Alsantali that have some degree of success include garlic gel, azelaic acid, topical onion juice, imiquimod, calcineurin inhibitors, botulinum toxin and photodynamic therapy.

An important element of the management of $A A$ is offering psychological support to stimulate increased self-esteem and adaptation to this disease. Some patients with AA require the support of a psychologist or psychiatrist. With psychological support and education of the patient about the disease, long-term improvement can be achieved [1,3].

It is also worth noting that when AA is limited to a single patch, the best option in most patients is leaving it untreated, as $80 \%$ of patients with single patches present for less than one year go into spontaneous remission [26]. 


\section{Conflict of interest}

There is no conflict of interest.

\section{References}

1. Brzezińska-Wcisło L, Lis-Święty A. Hair diseases - what's new? In: Dermatology - what's new? [Polish]. Vol. 1. Szepietowski J, Reich A (eds.). Cornetis, Wrocław 2009; 187-200.

2. Burgdorf WHC, Plewig G, Wolff HH, Landthaler M. Hair diseases. In: Braun-Falco Dermatology [Polish]. Vol. 2. Czelej, Lublin 2010; 1053-83.

3. Brzezińska-Wcisło L, Lis A, Kamińska G, Wcisło-Dziadecka D. Physiology and pathology of hair growth and loss on the human scalp [Polish]. Postep Derm Alergol 2003; 20: 260-6.

4. Brzezińska-Wcisło L, Wcisło-Dziadecka D, Meszyńska E, et al. New perspectives on the pathogenesis and treatment of hair disorders. Post Nauk Med 2012; 10: 800-5.

5. Łuczak M, Łuczak T, Cieścińska C, Czajkowski R. General treatment of alopecia areata [Polish]. Przegl Dermatol 2013; 100: 53-8.

6. Acikgöz G, Ozmen I, Cayirli M, et al. Pulse methylprednisolone therapy for the treatment of extensive alopecia areata. J Dermatolog Treat 2014; 25: 164-6.

7. Staumont-Sallé D, Vonarx M, Lengrand F, et al. Pulse corticosteroid therapy for alopecia areata: log-term outcome after 10. Dermatology 2012; 225: 81-7.

8. Bin Saif GA, Al-Khawajah MM, Al-Otaibi HM, et al. Efficacy and safety of oral mega pulse methylprednisolone for severe therapy resistant Alopecia areata. Sudi Med J 2012; 33: 284-91.

9. Alsantali A. Alopecia areata: a new treatment plan. Clin Cosmet Investig Dermatol 2011; 4: 107-15.

10. Farshi S, Mansouri P, Safar F, Khiabanloo SR. Could azathioprine be considered as a therapeutic alternative in the treatment of alopecia areata? A pilot study. Int I Dermatol 2010; 49: 1188-93.

11. Droitcourt C, Milpied B, Ezzedine K, et al. Interest of highdose pulse corticosteroid therapy combined with methotrexate for severe alopecia areata: a retrospective case series. Dermatology 2012; 224: 369-73.

12. Miteva M, Tosti A. Treatment options for alopecia: an update looking to the future. Expert Opin Pharmocter 2012; 13: 1271-81.

13. Bhat YJ, Manzoor S, Khan AR, Qayooni S. Trace element levels in alopecia areata. Indian J Dermatol Venereol Leprol 2009; 75: 29-31.

14. Ito T, Aoshima M, Ito N, et al. Combination therapy with oral PUVA and corticosteroid for recalcitrant alopecia areata. Arch Dermatol Res 2009; 301: 373-80.

15. Cho HR, Lew BL, Lew H, Sim WY. Treatment effects of intradermal botulinum toxin type $A$ injection on alopecia areata. Dermatol Surg 2010; 4: 2175-81.

16. Abedini H, Farshi S, Mirabzadeh A, Keshavarz S. Antidepressant effects of citalopram on treatment of alopecia areata in patients with major depressive disorder. J Dermatolog Treat 2014; 25: 153-5.

17. Otberg N. Systemic treatment for alopecia areata. Dermatol Ther 2011; 24: 320-5.

18. Luk NM, Chiu LS, Lee KC, et al. Efficacy and safety of diphenylcyclopropenone among Chinese patients with steroid resistant and extensive alopecia areata. J Eur Acad Dermatol Venereol 2013; 27: e400-5.
19. Campuzano-Maya G. Cure of alopecia areata after eradication of Helicobacter pylori: a new association? World J Gastroenterol 2011; 17: 3165-70.

20. Bayramgürler D, Demirsoy EO, Aktürk AS, Kiran R. Narrowband ultraviolet B phototherapy for alopecia areata. Photodermatol Photoimmunol Photomed 2011; 27: 325-7.

21. Raijiv M, Singh N. Bexarotene gel: a new topical therapy for alopecia areata. Int J Trichology 2010; 2: 66-7.

22. Ucak H, Kandi B, Cicek D, et al. The comparison of treatment with clobetasol propionate $0,05 \%$ and topical pimecrolimus $1 \%$ treatment in the treatment of alopecia areata. J Dermatolog Treat 2012; 23: 410-20.

23. Nasiri S, Haghpanah V, Taheri E, et al. Hair regrowth, with topical triiodothyronine ointment in patients with alopecia areata: a double-blind, randomized pilot clinical trial of efficacy. Eur Acad Dermatol Venereol 2012; 26: 654-6.

24. Manolache L, Petrescu-Seceleanu D, Benea V. Alopecia areata and relationship with stressful events in children. J Eur Acad Dermatol Venereol 2009; 23: 107-9.

25. Willemsen R, Vanderlinden J, Roseeuw D, Haentjens P. Increased history of childhood and lifetime traumatic events among adults with alopecia areata. J Eur Acad Dermatol Venereol 2009; 60: 388-93.

26. MacDonald Hull SP, Wood ML, Hutchinson PE, et al. British Association of Dermatologists. Guidelines for the management of alopecia areata. Br J Dermatol 2003; 149: 692-9. 